\title{
Tripled coincidence point theorems for a $\varphi$-contractive mapping in a complete metric space without the mixed $g$-monotone property
}

Phakdi Charoensawan*

"Correspondence: phakdi@hotmail.com Department of Mathematics, Faculty of Science, Chiang Mai University, Chiang Mai, 50200, Thailand

\section{第 Springer}

\begin{abstract}
In this paper, we show the existence of a tripled coincidence point theorem and a tripled common fixed point theorem for a $\varphi$-contractive mapping in a complete metric space without the mixed $g$-monotone property, using the concept of $(F, g$ )-invariant set. Further, we apply our results to the existence of a tripled coincidence point of the given mapping in a partially ordered metric space.
\end{abstract}

Keywords: tripled fixed point; tripled coincidence point; invariant set; mixed g-monotone; partially ordered set

\section{Introduction}

The existence of a fixed point for contraction type mappings in partially ordered metric spaces was first considered recently by Ran and Reurings [1] where they established some new results for contractions in partially ordered metric spaces and presented applications to matrix equations. Later, Nieto and Lopez [2,3] and Agarwal et al. [4] presented some new results for contractions in partially ordered metric spaces.

The concept of coupled fixed point was introduced by Guo and Lakshmikantham [5]. In 2006, Bhaskar and Lakshmikantham [6] introduced the concept of mixed monotone property for contractive operators of the form $F: X \times X \rightarrow X$, where $X$ is a partially ordered metric space. They also established coupled fixed point theorems for mappings which satisfy the mixed monotone property. After the publication of this work, many authors conducted research on the coupled fixed point theory in partially ordered metric spaces and different spaces. For example, see [7-19].

Later, Sintunavarat et al. $[15,16]$ proved the existence and uniqueness of coupled fixed point theorems for nonlinear contractions without the mixed monotone property and extended some coupled fixed point theorems of Bhaskar and Lakshmikantham [6] by using the concept of $F$-invariant set due to Samet and Vetro [10]. Recently, in 2013, Batra and Vashistha [17] introduced the concept of $(F, g)$-invariant set which is a generalization of an $F$-invariant set introduced by Samet and Vetro [10] and proved the existence coupled fixed point theorems for nonlinear contractions under $c$-distance in cone metric spaces having an $(F, g)$-invariant subset.

O2013 Charoensawan; licensee Springer. This is an Open Access article distributed under the terms of the Creative Commons Attribution License (http://creativecommons.org/licenses/by/2.0), which permits unrestricted use, distribution, and reproduction in any medium, provided the original work is properly cited. 
Tripled fixed point theorems for nonlinear mappings can be viewed as a generalization of coupled fixed point theorems and those can be applied to ODE problems. In 2011, Berinde and Borcut [20] introduced the concept of tripled fixed point for nonlinear mappings in partially ordered complete metric spaces and obtained existence and uniqueness theorems for contractive type mappings. Later, in 2012, Berinde and Borcut [21] introduced the concept of tripled coincidence point for a pair of nonlinear contractive mappings $F: X^{3} \rightarrow X$ and $g: X \rightarrow X$ and obtained tripled coincidence point theorems which generalized the results of [20]. After the publication of this work, the tripled fixed point and tripled coincidence point theory has been generalized in different directions in several spaces with applications by many mathematicians over the year (see $[11,12,22-$ $34])$.

Since 2011, some authors have studied triple fixed point and tripled coincidence point theory. This theory has received much attention in different spaces. Aydi et al. [30] established some tripled coincidence point results for a mixed $g$-monotone mapping $F$ : $X^{3} \rightarrow X$ satisfying $(\psi, \phi)$-contractions in ordered generalized metric spaces. Aydi et al. [27] introduced the concept of $W$-compatible mappings for mappings $F: X^{3} \rightarrow X$ and $g: X \rightarrow X$, where $(X, d)$ is an abstract metric space, and established some tripled coincidence and common tripled fixed point theorems in such spaces. Based on the notion of $W$ compatible, Aydi and Abbas [28] obtained tripled coincidence and common tripled fixed point results in the partial metric spaces which generalized and extended several wellknown comparable results in the existing literature. Afterward, Abbas et al. [23] proved the existence of tripled fixed point and tripled coincidence point theorems in intuitionistic fuzzy normed spaces. The results generalized and extended recent coupled fixed point theorems in intuitionistic fuzzy normed spaces.

On the other hand, a wide discussion on a tripled fixed point and a tripled coincidence point in partially ordered metric spaces has been dedicated to improvement and generalization. In 2011, Abbas et al. [22] obtained the existence of a tripled fixed point of multivalued nonlinear contraction mappings in partially ordered metric spaces. Amini-Harandi [11] introduced a new simple and unified approach to coupled and tripled fixed point theory and presented a new tripled fixed point result in partially ordered metric spaces. Later, Hussain et al. [12] introduced a new and simple approach to coupled and tripled coincidence point theory. They established coupled coincidence and tripled coincidence point results without any type of commutativity condition on $F$ and $g$.

One of the simplest ways to develop tripled fixed point and tripled coincidence point theory in partially ordered metric spaces is to consider contraction mappings. Using the concept of mixed monotone, Aydi et al. [25] discussed existence and uniqueness of some tripled fixed points for mappings having the mixed monotone property in partially ordered metric spaces by using Meir-Keeler type contractions. Furthermore, Aydi and Karapinar [26] proved some new Meir-Keeler type tripled fixed point theorems on partially ordered complete partial metric spaces. Aydi and Karapinar [29] established some triple fixed point theorems for mappings having the mixed monotone property in partially ordered metric spaces dependent on another contractive condition which is a generalization of the main results of Berinde and Borcut [20]. Charoensawan [33] proved the existence and uniqueness of a tripled fixed point involving a $(\varphi, \psi)$-contractive condition for mappings having the mixed monotone property in partially ordered metric spaces. 
In the case of tripled coincidence point theory, using the mixed $g$-monotone property, Borcut [31] established tripled coincidence point theorems for a pair of mappings $F: X^{3} \rightarrow X$ and $g: X \rightarrow X$ satisfying a nonlinear contractive condition and having the mixed $g$-monotone property in partially ordered metric spaces. The presented theorems extended existing results in the literature. Recently, Choudhury et al. [32] established some tripled coincidence point results in partially ordered metric spaces dependent on other contractions. Very recently, Aydi et al. [24] established tripled coincidence point theorems for a pair of mappings $F: X^{3} \rightarrow X$ and $g: X \rightarrow X$ satisfying weak $\phi$-contractions in partially ordered metric spaces. The results unified, generalized and complemented various known comparable results from Berinde and Borcut [21].

The purpose of this paper is to establish some tripled coincidence point theorems without the mixed $g$-monotone property by using the concept of $(F, g)$-invariant set in a complete metric space which are generalizations of the results of Aydi et al. [24].

\section{Preliminaries}

Let $(X, \leq)$ be a partially ordered set, and suppose that there is a metric $d$ on $X$ such that $(X, d)$ is a complete metric space. Consider on the product space $X \times X \times X$ the following partial order: for $(x, y, z),(u, v, w) \in X \times X \times X$,

$$
(u, v, w) \leq(x, y, z) \Longleftrightarrow x \geq u, \quad y \leq v, \quad z \geq w .
$$

Berinde and Borcut [21] introduced the concept of tripled coincidence point and studied existence and uniqueness theorems in partially ordered complete metric spaces.

Definition 2.1 ([21]) Let $(X, \leq)$ be a partially ordered set and two mappings $F: X \times X \times$ $X \rightarrow X, g: X \rightarrow X$. We say that $F$ has the mixed $g$-monotone property if for any $x, y, z \in X$,

$$
\begin{array}{llll}
x_{1}, x_{2} \in X, & g\left(x_{1}\right) \leq g\left(x_{2}\right) \quad \text { implies } & F\left(x_{1}, y, z\right) \leq F\left(x_{2}, y, z\right) \\
y_{1}, y_{2} \in X, & g\left(y_{1}\right) \leq g\left(y_{2}\right) \quad \text { implies } & F\left(x, y_{1}, z\right) \geq F\left(x, y_{2}, z\right)
\end{array}
$$

and

$$
z_{1}, z_{2} \in X, \quad g\left(z_{1}\right) \leq g\left(z_{2}\right) \quad \text { implies } \quad F\left(x, y, z_{1}\right) \leq F\left(x, y, z_{2}\right) .
$$

Definition 2.2 ([21]) An element $(x, y, z) \in X \times X \times X$ is called a tripled coincidence point of mappings $F$ and $g$ if $F(x, y, z)=g(x), F(y, x, y)=g(y)$ and $F(z, y, x)=g(z)$.

Definition 2.3 ([21]) Let $X$ be a nonempty set and $F: X \times X \times X \rightarrow X, g: X \rightarrow X$ be two mappings. We say $F$ and $g$ are commutative if $g(F(x, y, z))=F(g(x), g(y), g(z))$ for all $x, y, z \in X$.

Later, Aydi et al. [24] extended the tripled coincidence point theorems for a mixed $g$-monotone operator obtained by Berinde and Borcut [21]. For the sake of completeness, we recollect the main results of Aydi et al. [24] here.

Let the set of functions $\Phi=\left\{\varphi:[0,+\infty) \rightarrow[0,+\infty): \varphi(t)<t\right.$ and $\left.\lim _{r \rightarrow t^{+}} \varphi(r)<t, t>0\right\}$. 
Theorem 2.4 ([24]) Let $(X, \leq)$ be a partially ordered set, and suppose that there is a metric $d$ on $X$ such that $(X, d)$ is a complete metric space. Let $F: X \times X \times X \rightarrow X$ and $g: X \rightarrow X$ be such that $F$ has the mixed $g$-monotone property and $F\left(X^{3}\right) \subseteq g(X)$. Assume that there is a function $\varphi \in \Phi$ such that

$$
\begin{aligned}
& d(F(x, y, z), F(u, v, w))+d(F(y, x, y), F(v, u, v))+d(F(z, y, x), F(w, v, u)) \\
& \quad \leq 3 \varphi\left(\frac{d(g(x), g(u))+d(g(y), g(v))+d(g(z), g(w))}{3}\right)
\end{aligned}
$$

for all $x, y, z, u, v, w \in X$ with $g(x) \geq g(u), g(y) \leq g(v)$ and $g(z) \geq g(w)$. Assume that $F$ is continuous, $g$ is continuous and commutes with $F$.

If there exist $x_{0}, y_{0}, z_{0} \in X$ such that

$$
g\left(x_{0}\right) \leq F\left(x_{0}, y_{0}, z_{0}\right), \quad g\left(y_{0}\right) \geq F\left(y_{0}, x_{0}, y_{0}\right) \quad \text { and } \quad g\left(z_{0}\right) \leq F\left(z_{0}, y_{0}, x_{0}\right),
$$

then there exist $x, y, z \in X$ such that

$$
g(x)=F(x, y, z), \quad g(y)=F(y, x, y) \quad \text { and } \quad g(z)=F(z, y, x) .
$$

Definition 2.5 ([24]) Let $(X, \leq)$ be a partially ordered set and $d$ be a metric on $X$. We say that $(X, d, \leq)$ is regular if the following conditions hold:

(i) if a non-decreasing sequence $\left\{x_{n}\right\} \rightarrow x$ in $X$, then $x_{n} \leq x$ for all $n$,

(ii) if a non-increasing sequence $\left\{y_{n}\right\} \rightarrow y$ in $X$, then $y \leq y_{n}$ for all $n$.

Theorem 2.6 ([24]) Let $(X, \leq)$ be a partially ordered set, and suppose that there is a metric $d$ on $X$ such that $(X, d, \leq)$ is regular. Suppose that there exist $\varphi \in \Phi$ and mapping $F: X \times$ $X \times X \rightarrow X$ and $g: X \rightarrow X$ are such that (5) hold for any $x, y, z, u, v, w \in X$ with $g(x) \geq$ $g(u), g(y) \leq g(v)$ and $g(z) \geq g(w)$. Suppose also that $(g(X), d)$ is complete, $F$ has the mixed g-monotone property and $F\left(X^{3}\right) \subseteq g(X)$.

If there exist $x_{0}, y_{0}, z_{0} \in X$ such that

$$
g\left(x_{0}\right) \leq F\left(x_{0}, y_{0}, z_{0}\right), \quad g\left(y_{0}\right) \geq F\left(y_{0}, x_{0}, y_{0}\right) \quad \text { and } \quad g\left(z_{0}\right) \leq F\left(z_{0}, y_{0}, x_{0}\right),
$$

then there exist $x, y, z \in X$ such that

$$
g(x)=F(x, y, z), \quad g(y)=F(y, x, y) \quad \text { and } \quad g(z)=F(z, y, x) .
$$

Batra and Vashistha [17] introduced an $(F, g)$-invariant set which is a generalization of an $F$-invariant set introduced by Samet and Vetro [10].

Definition 2.7 ([13]) Let $(X, \leq)$ be a metric space and $F: X \times X \rightarrow X, g: X \rightarrow X$ be given mappings. Let $M$ be a nonempty subset of $X^{4}$. We say that $M$ is an $(F, g)$-invariant subset of $X^{4}$ if and only if, for all $x, y, z, w \in X$,

(i) $(x, y, z, w) \in M \Leftrightarrow(w, z, y, x) \in M$,

(ii) $(g(x), g(y), g(z), g(w)) \in M \Rightarrow(F(x, y), F(y, x), F(z, w), F(w, z)) \in M$. 
In this article, we establish tripled coincidence point theorems for $F: X \times X \times X \rightarrow X$ and $g: X \rightarrow X$ satisfying nonlinear contractive conditions without the mixed $g$-monotone property by using the concept of $(F, g)$-invariant set in complete metric spaces.

\section{Main results}

We give the notions of $F$-invariant set and $(F, g)$-invariant set which are useful for main results.

Definition 3.1 Let $(X, \leq)$ be a metric space and $F: X \times X \times X \rightarrow X$ be a given mapping. Let $M$ be a nonempty subset of $X^{6}$. We say that $M$ is an F-invariant subset of $X^{6}$ if and only if, for all $x, y, z, u, v, w \in X$,

$$
\begin{aligned}
& (x, y, z, u, v, w) \in M \\
& \quad \Rightarrow \quad(F(x, y, z), F(y, x, y), F(z, y, x), F(u, v, w), F(v, u, v), F(w, u, v)) \in M .
\end{aligned}
$$

Definition 3.2 Let $(X, \leq)$ be a metric space and $M$ be a subset of $X^{6}$. We say that $M$ satisfies the transitive property if and only if, for all $x, y, z, u, v, w, a, b, c \in X$,

$$
(x, y, z, u, v, w) \in M \quad \text { and } \quad(u, v, w, a, b, c) \in M \quad \Rightarrow \quad(x, y, z, a, b, c) \in M .
$$

Definition 3.3 Let $(X, \leq)$ be a metric space and $F: X \times X \times X \rightarrow X, g: X \rightarrow X$ be given mappings. Let $M$ be a nonempty subset of $X^{6}$. We say that $M$ is an $(F, g)$-invariant subset of $X^{6}$ if and only if, for all $x, y, z, u, v, w \in X$,

$$
\begin{aligned}
& (g(x), g(y), g(z), g(u), g(v), g(w)) \in M \\
& \quad \Rightarrow \quad(F(x, y, z), F(y, x, y), F(z, y, x), F(u, v, w), F(v, u, v), F(w, u, v)) \in M .
\end{aligned}
$$

\section{Remark 3.4}

1. The set $M=X^{6}$ is trivially $(F, g)$-invariant, which satisfies the transitive property.

2. Every $F$-invariant set is $\left(F, I_{X}\right)$-invariant, when $I_{X}$ denotes an identity map on $X$.

Example 3.5 Let $(X, \leq)$ be a partially ordered set, and suppose that there is a metric $d$ on $X$ such that $(X, d)$ is a complete metric space. Let $F: X \times X \times X \rightarrow X$ and $g: X \rightarrow X$ be two mappings such that $F$ satisfies mixed $g$-monotone property. Define a subset $M \subseteq X^{6}$ by $M=\left\{(a, b, c, d, e, f) \in X^{6}: a \geq d, b \leq e, c \geq f\right\}$. Then $M$ is an $F$-invariant and $(F, g)$-invariant subset of $X^{6}$, which satisfies the transitive property.

The following example shows that we may have an $(F, g)$-invariant set which is not $F$-invariant set.

Example 3.6 Let $X=\mathbb{R}$ and $F: X \times X \times X \rightarrow X$ be defined by $F(x, y, z)=1-x^{2}$. Let $g: X \rightarrow$ $X$ be given by $g(x)=x-1$. Then it is easy to show that $M=\left\{(x, 0, z, u, 0, w) \in X^{6}: x=z, u=\right.$ $w\}$ is an $(F, g)$-invariant subset of $X^{6}$ but not an $F$-invariant subset of $X^{6}$ as $(1,0,1,1,0,1) \in$ $M$, but $(F(1,0,1), F(0,1,0), F(1,0,1), F(1,0,1), F(0,0,0), F(1,0,1))=(0,1,0,0,1,0) \notin M$.

Our first main result is the following. 
Theorem 3.7 Let $(X, \leq)$ be a complete metric space and $M$ be a nonempty subset of $X^{6}$. Assume that there is a function $\varphi:[0,+\infty) \rightarrow[0,+\infty)$ with $0=\varphi(0)<\varphi(t)<t$ and $\lim _{r \rightarrow t^{+}} \varphi(r)<t$ for each $t>0$, and also suppose that $F: X \times X \times X \rightarrow X$ and $g: X \rightarrow X$ are two continuous functions such that

$$
\begin{aligned}
& d(F(x, y, z), F(u, v, w))+d(F(y, x, y), F(v, u, v))+d(F(z, y, x), F(w, v, u)) \\
& \quad \leq 3 \varphi\left(\frac{d(g(x), g(u))+d(g(y), g(v))+d(g(z), g(w))}{3}\right)
\end{aligned}
$$

for all $x, y, z, u, v, w \in X$ with $(g(x), g(y), g(z), g(u), g(v), g(w)) \in M$ or $(g(u), g(v), g(w), g(x)$, $g(y), g(z)) \in M$. Suppose that $F(X \times X \times X) \subseteq g(X), g$ commutes with $F$.

If there exists $\left(x_{0}, y_{0}, z_{0}\right) \in X \times X \times X$ such that

$$
\left(F\left(x_{0}, y_{0}, z_{0}\right), F\left(y_{0}, x_{0}, y_{0}\right), F\left(z_{0}, y_{0}, x_{0}\right), g\left(x_{0}\right), g\left(y_{0}\right), g\left(z_{0}\right)\right) \in M
$$

and $M$ is an $(F, g)$-invariant set which satisfies the transitive property, then there exist $x, y, z \in X$ such that

$$
g(x)=F(x, y, z), \quad g(y)=F(y, x, y) \quad \text { and } \quad g(z)=F(z, y, x) .
$$

Proof Let $\left(x_{0}, y_{0}, z_{0}\right) \in X \times X \times X$ satisfy $\left(F\left(x_{0}, y_{0}, z_{0}\right), F\left(y_{0}, x_{0}, y_{0}\right), F\left(z_{0}, y_{0}, x_{0}\right), g\left(x_{0}\right), g\left(y_{0}\right)\right.$, $\left.g\left(z_{0}\right)\right) \in M$. Since $F(X \times X \times X) \subseteq g(X)$, we can choose $x_{1}, y_{1}, z_{1} \in X$ such that $g\left(x_{1}\right)=$ $F\left(x_{0}, y_{0}, z_{0}\right), g\left(y_{1}\right)=F\left(y_{0}, x_{0}, y_{0}\right)$ and $g\left(z_{1}\right)=F\left(z_{0}, y_{0}, x_{0}\right)$. Again from $F(X \times X \times X) \subseteq g(X)$ we can choose $x_{2}, y_{2}, z_{2} \in X$ such that $g\left(x_{2}\right)=F\left(x_{1}, y_{1}, z_{1}\right), g\left(y_{2}\right)=F\left(y_{1}, x_{1}, y_{1}\right)$ and $g\left(z_{2}\right)=$ $F\left(z_{1}, y_{1}, x_{1}\right)$.

Continuing this process, we can construct sequences $\left\{g\left(x_{n}\right)\right\},\left\{g\left(y_{n}\right)\right\}$ and $\left\{g\left(z_{n}\right)\right\}$ in $X$ such that

$$
\begin{gathered}
g\left(x_{n}\right)=F\left(x_{n-1}, y_{n-1}, z_{n-1}\right), \quad g\left(y_{n}\right)=F\left(y_{n-1}, x_{n-1}, y_{n-1}\right) \\
\text { and } g\left(z_{n}\right)=F\left(z_{n-1}, y_{n-1}, x_{n-1}\right) \quad \text { for all } n \geq 1 .
\end{gathered}
$$

If there exists $k \in N$ such that $g\left(x_{k}\right)=g\left(x_{k-1}\right), g\left(y_{k}\right)=g\left(y_{k-1}\right)$ and $g\left(z_{k}\right)=g\left(z_{k-1}\right)$, then $g\left(x_{k}\right)=g\left(x_{k-1}\right)=F\left(x_{k-1}, y_{k-1}, z_{k-1}\right), g\left(y_{k}\right)=g\left(y_{k-1}\right)=F\left(y_{k-1}, x_{k-1}, y_{k-1}\right)$ and $g\left(z_{k}\right)=g\left(z_{k-1}\right)=$ $F\left(z_{k-1}, y_{k-1}, x_{k-1}\right)$. Thus, $\left(x_{k-1}, y_{k-1}, z_{k-1}\right)$ is a tripled coincidence point of $F$. This finishes the proof.

Therefore, we may assume that $g\left(x_{k}\right) \neq g\left(x_{k-1}\right)$ or $g\left(y_{k}\right) \neq g\left(y_{k-1}\right)$, or $g\left(z_{k}\right) \neq g\left(z_{k-1}\right)$ for all $n \geq 1$. Since $\left(F\left(x_{0}, y_{0}, z_{0}\right), F\left(y_{0}, x_{0}, y_{0}\right), F\left(z_{0}, y_{0}, x_{0}\right), g\left(x_{0}\right), g\left(y_{0}\right), g\left(z_{0}\right)\right)=\left(g\left(x_{1}\right), g\left(y_{1}\right), g\left(z_{1}\right)\right.$, $\left.g\left(x_{0}\right), g\left(y_{0}\right), g\left(z_{0}\right)\right) \in M$ and $M$ is an $(F, g)$-invariant set, we have $\left(F\left(x_{1}, y_{1}, z_{1}\right), F\left(y_{1}, x_{1}, y_{1}\right)\right.$, $\left.F\left(z_{1}, y_{1}, x_{1}\right), F\left(x_{0}, y_{0}, z_{0}\right), F\left(y_{0}, x_{0}, y_{0}\right), F\left(z_{0}, y_{0}, x_{0}\right)\right)=\left(g\left(x_{2}\right), g\left(y_{2}\right), g\left(z_{2}\right), g\left(x_{1}\right), g\left(y_{1}\right), g\left(z_{1}\right)\right) \in M$.

By repeating this argument, we get $\left(F\left(x_{n-1}, y_{n-1}, z_{n-1}\right), F\left(y_{n-1}, x_{n-1}, y_{n-1}\right), F\left(z_{n-1}, y_{n-1}, x_{n-1}\right)\right.$, $\left.F\left(x_{n}, y_{n}, z_{n}\right), F\left(y_{n}, x_{n}, y_{n}\right), F\left(z_{n}, y_{n}, x_{n}\right)\right)=\left(g\left(x_{n}\right), g\left(y_{n}\right), g\left(z_{n}\right), g\left(x_{n-1}\right), g\left(y_{n-1}\right), g\left(z_{n-1}\right)\right) \in M$ for all $n \geq 1$.

Consider now the sequence of nonnegative real numbers $\left\{\delta_{n}\right\}_{n=1}^{\infty}$ given by

$$
\delta_{n-1}=d\left(g\left(x_{n}\right), g\left(x_{n-1}\right)\right)+d\left(g\left(y_{n}\right), g\left(y_{n-1}\right)\right)+d\left(g\left(z_{n}\right), g\left(z_{n-1}\right)\right), \quad n \geq 1 .
$$


Since $\left(g\left(x_{n}\right), g\left(y_{n}\right), g\left(z_{n}\right), g\left(x_{n-1}\right), g\left(y_{n-1}\right), g\left(z_{n-1}\right)\right) \in M$, we have

$$
\begin{aligned}
d( & \left.F\left(x_{n}, y_{n}, z_{n}\right), F\left(x_{n-1}, y_{n-1}, z_{n-1}\right)\right)+d\left(F\left(y_{n}, x_{n}, y_{n}\right), F\left(y_{n-1}, x_{n-1}, y_{n-1}\right)\right) \\
& +d\left(F\left(z_{n}, y_{n}, x_{n}\right), F\left(z_{n-1}, y_{n-1}, x_{n-1}\right)\right) \\
= & d\left(g\left(x_{n+1}\right), g\left(x_{n}\right)\right)+d\left(g\left(y_{n+1}\right), g\left(y_{n}\right)\right)+d\left(g\left(z_{n+1}\right), g\left(z_{n}\right)\right) \\
= & \delta_{n} .
\end{aligned}
$$

The right-hand side of (6) will be equal to

$$
3 \varphi\left(\frac{d\left(g\left(x_{n}\right), g\left(x_{n-1}\right)\right)+d\left(g\left(y_{n}\right), g\left(y_{n-1}\right)\right)+d\left(g\left(z_{n}\right), g\left(z_{n-1}\right)\right)}{3}\right)=3 \varphi\left(\frac{\delta_{n-1}}{3}\right) .
$$

Therefore, the sequence $\left\{\delta_{n}\right\}_{n=1}^{\infty}$ satisfies

$$
\delta_{n} \leq 3 \varphi\left(\frac{\delta_{n-1}}{3}\right)<\delta_{n-1} \quad \text { for all } n \geq 1
$$

From (10) it follows that the sequence $\left\{\delta_{n}\right\}_{n=1}^{\infty}$ is decreasing. Therefore, there exists some $\delta \geq 0$ such that

$$
\begin{aligned}
\lim _{n \rightarrow \infty} \delta_{n} & =\lim _{n \rightarrow \infty}\left[d\left(g\left(x_{n+1}\right), g\left(x_{n}\right)\right)+d\left(g\left(y_{n+1}\right), g\left(y_{n}\right)\right)+d\left(g\left(z_{n+1}\right), g\left(z_{n}\right)\right)\right] \\
& =\delta .
\end{aligned}
$$

We shall prove that $\delta=0$. Assume, to the contrary, that $\delta>0$. Then, by letting $n \rightarrow \infty$ in (10), from $0=\varphi(0)<\varphi(t)<t$ and $\lim _{r \rightarrow t^{+}} \varphi(r)<t$, for each $t>0$, we have

$$
\delta=\lim _{n \rightarrow \infty} \delta_{n} \leq 3 \lim _{n \rightarrow \infty} \varphi\left(\frac{\delta_{n-1}}{3}\right)=3 \lim _{\delta_{n-1} \rightarrow \delta^{+}} \varphi\left(\frac{\delta_{n-1}}{3}\right)<3 \frac{\delta}{3}=\delta
$$

a contradiction. Thus $\delta=0$ and hence

$$
\lim _{n \rightarrow \infty} \delta_{n}=\lim _{n \rightarrow \infty}\left[d\left(g\left(x_{n+1}\right), g\left(x_{n}\right)\right)+d\left(g\left(y_{n+1}\right), g\left(y_{n}\right)\right)+d\left(g\left(z_{n+1}\right), g\left(z_{n}\right)\right)\right]=0
$$

We now prove that $\left\{g\left(x_{n}\right)\right\},\left\{g\left(y_{n}\right)\right\}$ and $\left\{g\left(z_{n}\right)\right\}$ are Cauchy sequences in $(X, d)$. Suppose, to the contrary, that at least one of $\left\{g\left(x_{n}\right)\right\},\left\{g\left(y_{n}\right)\right\}$ or $\left\{g\left(z_{n}\right)\right\}$ is not a Cauchy sequence. Then there exists $\varepsilon>0$ for which we can find subsequences $\left\{g\left(x_{m(k)}\right)\right\}$ and $\left\{g\left(x_{n(k)}\right)\right\}$ of $\left\{g\left(x_{n}\right)\right\}$, $\left\{g\left(y_{m(k)}\right)\right\}$ and $\left\{g\left(y_{n(k)}\right)\right\}$ of $\left\{g\left(y_{n}\right)\right\}$ and $\left\{g\left(z_{m(k)}\right)\right\}$ and $\left\{g\left(z_{n(k)}\right)\right\}$ of $\left\{g\left(z_{n}\right)\right\}$ with $n(k)>m(k) \geq K$ such that

$$
d\left(g\left(x_{n(k)}\right), g\left(x_{m(k)}\right)\right)+d\left(g\left(y_{n(k)}\right), g\left(y_{m(k)}\right)\right)+d\left(g\left(z_{n(k)}\right), g\left(z_{m(k)}\right)\right) \geq \varepsilon, \quad k \in \mathbb{N} .
$$

Further, corresponding to $m(k)$, we can choose $n(k)$ in such a way that it is the smallest integer with $n(k)>m(k) \geq K$ and satisfies (14). Then

$$
d\left(g\left(x_{n(k)-1}\right), g\left(x_{m(k)}\right)\right)+d\left(g\left(y_{n(k)-1}\right), g\left(y_{m(k)}\right)\right)+d\left(g\left(z_{n(k)-1}\right), g\left(z_{m(k)}\right)\right)<\varepsilon .
$$


Using (14) and (15) and the triangle inequality, we have

$$
\begin{aligned}
\varepsilon \leq & r_{k}:=d\left(g\left(x_{n(k)}\right), g\left(x_{m(k}\right)\right)+d\left(g\left(y_{n(k)}\right), g\left(y_{m(k)}\right)\right)+d\left(g\left(z_{n(k)}\right), g\left(z_{m(k)}\right)\right) \\
\leq & d\left(g\left(x_{n(k)}\right), g\left(x_{n(k)-1}\right)\right)+d\left(g\left(x_{n(k)-1}\right), g\left(x_{m(k)}\right)\right) \\
& +d\left(g\left(y_{n(k)}\right), g\left(y_{n(k)-1}\right)\right)+d\left(g\left(y_{n(k)-1}\right), g\left(y_{m(k)}\right)\right) \\
& +d\left(g\left(z_{n(k)}\right), g\left(z_{n(k)-1}\right)\right)+d\left(g\left(z_{n(k)-1}\right), g\left(z_{m(k)}\right)\right) \\
\leq & d\left(g\left(x_{n(k)}\right), g\left(x_{n(k)-1}\right)\right)+d\left(g\left(y_{n(k)}\right), g\left(y_{n(k)-1}\right)\right)+d\left(g\left(z_{n(k)}\right), g\left(z_{n(k)-1}\right)\right) \\
& +\varepsilon
\end{aligned}
$$

Letting $k \rightarrow \infty$ in the above inequality and using (13), we get

$$
\begin{aligned}
\varepsilon & \leq \lim _{k \rightarrow \infty} r_{k} \\
& \leq \lim _{k \rightarrow \infty}\left[d\left(g\left(x_{n(k)}\right), g\left(x_{n(k)-1}\right)\right)+d\left(g\left(y_{n(k)}\right), g\left(y_{n(k)-1}\right)\right)+d\left(g\left(z_{n(k)}\right), g\left(z_{n(k)-1}\right)\right)+\varepsilon\right]=\varepsilon,
\end{aligned}
$$

that is,

$$
\begin{aligned}
\lim _{k \rightarrow \infty} r_{k} & =\lim _{k \rightarrow \infty}\left[d\left(g\left(x_{n(k)}\right), g\left(x_{m(k}\right)\right)+d\left(g\left(y_{n(k)}\right), g\left(y_{m(k)}\right)\right)+d\left(g\left(z_{n(k)}\right), g\left(z_{m(k)}\right)\right)\right] \\
& =\varepsilon .
\end{aligned}
$$

Since $n(k)>m(k)$ and $M$ satisfies the transitive property, we get

$$
\left(g\left(x_{n(k)}\right), g\left(y_{n(k)}\right), g\left(z_{n(k)}\right), g\left(x_{m(k)}\right), g\left(y_{m(k)}\right), g\left(z_{m(k)}\right)\right) \in M
$$

Hence we can use (6) to obtain

$$
\begin{aligned}
d( & \left.g\left(x_{n(k)+1}\right), g\left(x_{m(k)+1}\right)\right)+d\left(g\left(y_{n(k)+1}\right), g\left(y_{m(k)+1}\right)\right)+d\left(g\left(z_{n(k)+1}\right), g\left(z_{m(k)+1}\right)\right) \\
= & d\left(F\left(x_{n(k)}, y_{n(k)}, z_{n(k)}\right), F\left(x_{m(k)}, y_{m(k)}, z_{m(k)}\right)\right) \\
& +d\left(F\left(y_{n(k)}, x_{n(k)}, y_{n(k)}\right), F\left(y_{m(k)}, x_{m(k)}, y_{m(k)}\right)\right) \\
& +d\left(F\left(z_{n(k)}, y_{n(k)}, x_{n(k)}\right), F\left(z_{m(k)}, y_{m(k)}, x_{m(k)}\right)\right) \\
\leq & 3 \varphi\left(\frac{d\left(g\left(x_{n(k)}\right), g\left(x_{m(k)}\right)\right)+d\left(g\left(y_{n(k)}\right), g\left(y_{m(k)}\right)\right)+d\left(g\left(z_{n(k)}\right), g\left(z_{m(k)}\right)\right)}{3}\right) \\
= & 3 \varphi\left(\frac{r_{k}}{3}\right) .
\end{aligned}
$$

By (18), we have

$$
r_{k+1} \leq 3 \varphi\left(\frac{r_{k}}{3}\right)
$$

Letting $k \rightarrow \infty$ in (19) and using (13) and (17) and $\lim _{r \rightarrow t^{+}} \varphi(r)<t$ for each $t>0$, we have

$$
\varepsilon=\lim _{k \rightarrow \infty} r_{k} \leq 3 \lim _{k \rightarrow \infty} \varphi\left(\frac{r_{k}}{3}\right)=3 \lim _{r_{k} \rightarrow \varepsilon^{+}} \varphi\left(\frac{r_{k}}{3}\right)<2 \frac{\varepsilon}{3}=\varepsilon,
$$


which is a contradiction. This shows that $\left\{g\left(x_{n}\right)\right\},\left\{g\left(y_{n}\right)\right\}$ and $\left\{g\left(z_{n}\right)\right\}$ are Cauchy sequences. Since $X$ is a complete metric space, there exist $x, y, z \in X$ such that

$$
\lim _{n \rightarrow \infty} g\left(x_{n}\right)=x, \quad \lim _{n \rightarrow \infty} g\left(y_{n}\right)=y \quad \text { and } \quad \lim _{n \rightarrow \infty} g\left(z_{n}\right)=z .
$$

From (20) and continuity of $g$,

$$
\lim _{n \rightarrow \infty} g\left(g\left(x_{n}\right)\right)=g(x), \quad \lim _{n \rightarrow \infty} g\left(g\left(y_{n}\right)\right)=g(y) \quad \text { and } \quad \lim _{n \rightarrow \infty} g\left(g\left(z_{n}\right)\right)=g(z) .
$$

From (7) and commutativity of $F$ and $g$,

$$
\begin{aligned}
& g\left(g\left(x_{n+1}\right)\right)=g\left(F\left(x_{n}, y_{n}, z_{n}\right)\right)=F\left(g\left(x_{n}\right), g\left(y_{n}\right), g\left(z_{n}\right)\right), \\
& g\left(g\left(y_{n+1}\right)\right)=g\left(F\left(y_{n}, x_{n}, y_{n}\right)\right)=F\left(g\left(y_{n}\right), g\left(x_{n}\right), g\left(y_{n}\right)\right), \\
& g\left(g\left(z_{n+1}\right)\right)=g\left(F\left(z_{n}, y_{n}, x_{n}\right)\right)=F\left(g\left(z_{n}\right), g\left(y_{n}\right), g\left(x_{n}\right)\right) .
\end{aligned}
$$

We now show that $g(x)=F(x, y, z), g(y)=F(y, x, y)$ and $g(z)=F(z, y, x)$. Taking the limit as $n \rightarrow \infty$ in (22), (23) and (24), by (20), (21), continuity of $F$ and commutativity of $F$ and $g$, we get

$$
\begin{aligned}
g(x) & =g\left(\lim _{n \rightarrow \infty} g\left(x_{n+1}\right)\right)=\lim _{n \rightarrow \infty} g\left(g\left(x_{n+1}\right)\right)=\lim _{n \rightarrow \infty} g\left(F\left(x_{n}, y_{n}, z_{n}\right)\right) \\
& =\lim _{n \rightarrow \infty} F\left(g\left(x_{n}\right), g\left(y_{n}\right), g\left(z_{n}\right)\right)=F\left(\lim _{n \rightarrow \infty} g\left(x_{n}\right), \lim _{n \rightarrow \infty} g\left(y_{n}\right), \lim _{n \rightarrow \infty} g\left(z_{n}\right)\right) \\
& =F(x, y, z), \\
g(y) & =g\left(\lim _{n \rightarrow \infty} g\left(y_{n+1}\right)\right)=\lim _{n \rightarrow \infty} g\left(g\left(y_{n+1}\right)\right)=\lim _{n \rightarrow \infty} g\left(F\left(y_{n}, x_{n}, y_{n}\right)\right) \\
& =\lim _{n \rightarrow \infty} F\left(g\left(y_{n}\right), g\left(x_{n}\right), g\left(y_{n}\right)\right)=F\left(\lim _{n \rightarrow \infty} g\left(y_{n}\right), \lim _{n \rightarrow \infty} g\left(x_{n}\right), \lim _{n \rightarrow \infty} g\left(y_{n}\right)\right) \\
& =F(y, x, y)
\end{aligned}
$$

and

$$
\begin{aligned}
g(z) & =g\left(\lim _{n \rightarrow \infty} g\left(z_{n+1}\right)\right)=\lim _{n \rightarrow \infty} g\left(g\left(z_{n+1}\right)\right)=\lim _{n \rightarrow \infty} g\left(F\left(z_{n}, y_{n}, x_{n}\right)\right) \\
& =\lim _{n \rightarrow \infty} F\left(g\left(z_{n}\right), g\left(y_{n}\right), g\left(x_{n}\right)\right)=F\left(\lim _{n \rightarrow \infty} g\left(z_{n}\right), \lim _{n \rightarrow \infty} g\left(y_{n}\right), \lim _{n \rightarrow \infty} g\left(x_{n}\right)\right) \\
& =F(z, y, x) .
\end{aligned}
$$

Thus we prove that $g(x)=F(x, y, z), g(y)=F(y, x, y)$ and $g(z)=F(z, y, x)$.

Theorem 3.8 Let $(X, d)$ be a complete metric space and $M$ be a nonempty subset of $X^{6}$. Assume that there is a function $\varphi:[0, \infty) \rightarrow[0, \infty)$ with $0=\varphi(0)<\varphi(t)<t$ and $\lim _{r \rightarrow t^{+}} \varphi(t)<$ $t$ for each $t>0$, and also suppose that $F: X \times X \times X \rightarrow X$ and $g: X \rightarrow X$ are two continuous functions such that

$$
\begin{aligned}
& d(F(x, y, z), F(u, v, w))+d(F(y, x, y), F(v, u, v))+d(F(z, y, x), F(w, v, u)) \\
& \quad \leq 3 \varphi\left(\frac{d(g(x), g(u))+d(g(y), g(v))+d(g(z), g(w))}{3}\right)
\end{aligned}
$$


for all $x, y, z, u, v, w \in X$ with $(g(x), g(y), g(z), g(u), g(v), g(w)) \in M$ or $(g(u), g(v), g(w), g(x)$, $g(y), g(z)) \in M$. Suppose that $F(X \times X \times X) \subseteq g(X)$, $g$ commutes with $F$ and $(g(X), d)$ is a complete metric space and for any three sequences $\left\{x_{n}\right\},\left\{y_{n}\right\},\left\{z_{n}\right\}$ with $\left(x_{n+1}, y_{n+1}, z_{n+1}, x_{n}, y_{n}\right.$, $\left.z_{n}\right) \in M,\left\{x_{n}\right\} \rightarrow x,\left\{y_{n}\right\} \rightarrow y$ and $\left\{z_{n}\right\} \rightarrow z$ for all $n \geq 1$ imply $\left(x, y, z, x_{n}, y_{n}, z_{n}\right) \in M$ for all $n \geq 1$.If there exists $\left(x_{0}, y_{0}, z_{0}\right) \in X \times X \times X$ such that $\left(F\left(x_{0}, y_{0}, z_{0}\right), F\left(y_{0}, x_{0}, y_{0}\right), F\left(z_{0}, y_{0}, x_{0}\right)\right.$, $\left.g\left(x_{0}\right), g\left(y_{0}\right), g\left(z_{0}\right)\right) \in M$ and $M$ is an $(F, g)$-invariant set which satisfies the transitive property, then there exist $x, y, z \in X$ such that

$$
g(x)=F(x, y, z), \quad g(y)=F(y, x, y) \quad \text { and } \quad g(z)=F(z, y, x) .
$$

Proof Consider the Cauchy sequences $\left\{g\left(x_{n}\right)\right\},\left\{g\left(y_{n}\right)\right\}$ and $\left\{g\left(z_{n}\right)\right\}$ as in the proof of Theorem 3.7. Since $(g(X), d)$ is a complete metric space, there exist $x, y, z \in X$ such that $\left\{g\left(x_{n}\right)\right\} \rightarrow g(x),\left\{g\left(y_{n}\right)\right\} \rightarrow g(y)$ and $\left\{g\left(z_{n}\right)\right\} \rightarrow g(z)$. By assumption, we have $(g(x), g(y), g(z)$, $\left.g\left(x_{n}\right), g\left(y_{n}\right), g\left(z_{n}\right)\right) \in M$ for all $n \geq 1$; by the triangle inequality and (6), we get

$$
\begin{aligned}
& d(F(x, y, z), g(x))+d(F(y, x, y), g(y))+d(F(z, y, x), g(z)) \\
& \leq \\
& \quad d\left(F(x, y, z), g\left(x_{n+1}\right)\right)+d\left(g\left(x_{n+1}\right), g(x)\right) \\
& \quad+d\left(F(y, x, y), g\left(y_{n+1}\right)\right)+d\left(g\left(y_{n+1}\right), g(y)\right) \\
& \quad+d\left(F(z, y, x), g\left(z_{n+1}\right)\right)+d\left(g\left(z_{n+1}\right), g(z)\right) \\
& =d\left(F(x, y, z), F\left(x_{n}, y_{n}, z_{n}\right)\right)+d\left(g\left(x_{n+1}\right), g(x)\right) \\
& \quad+d\left(F(y, x, y), F\left(y_{n}, x_{n}, y_{n}\right)\right)+d\left(g\left(y_{n+1}\right), g(y)\right) \\
& \quad+d\left(F(z, y, x), F\left(z_{n}, y_{n}, x_{n}\right)\right)+d\left(g\left(z_{n+1}\right), g(z)\right) \\
& \leq 3 \varphi\left(\frac{d\left(g\left(x_{n}\right), g(x)\right)+d\left(g\left(y_{n}\right), g(y)\right)+d\left(g\left(z_{n}\right), g(z)\right)}{3}\right) \\
& \quad+d\left(g\left(x_{n+1}\right), g(x)\right)+d\left(g\left(y_{n+1}\right), g(y)\right)+d\left(g\left(z_{n+1}\right), g(z)\right) .
\end{aligned}
$$

Taking the limit as $n \rightarrow \infty$ in the inequality above and using the fact that $\lim _{r \rightarrow 0^{+}} \varphi(0)=0$, we obtain

$$
d(F(x, y, z), g(x))+d(F(y, x, y), g(y))+d(F(z, y, x), g(z))=0,
$$

which implies that $g(x)=F(x, y, z), g(y)=F(y, x, y)$ and $g(z)=F(z, y, x)$.

Example 3.9 Let $X=\mathbb{R}, d(x, y)=|x-y|$ and $F: X \times X \times X \rightarrow X$ be defined by

$$
F(x, y, z)=\frac{4 x+y+2 z}{24}, \quad(x, y, z) \in X^{3} .
$$

The mapping $F$ does not satisfy the mixed $g$-monotone property.

Let $M=X^{6}$ and $(x, y, z, u, v, w) \in M$, we have

$$
\begin{aligned}
& \left|\frac{4 x+y+2 z}{24}-\frac{4 u+v+2 w}{24}\right| \leq\left|\frac{x-u}{6}\right|+\left|\frac{y-v}{24}\right|+\left|\frac{z-w}{12}\right|, \\
& \left|\frac{4 y+x+2 y}{24}-\frac{4 v+u+2 v}{24}\right| \leq\left|\frac{y-v}{6}\right|+\left|\frac{x-u}{24}\right|+\left|\frac{y-v}{12}\right|
\end{aligned}
$$


and

$$
\left|\frac{4 z+y+2 x}{24}-\frac{4 w+v+2 u}{24}\right| \leq\left|\frac{z-w}{6}\right|+\left|\frac{y-v}{24}\right|+\left|\frac{x-u}{12}\right|
$$

We get

$$
\begin{aligned}
& \left|\frac{4 x+y+2 z}{24}-\frac{4 u+v+2 w}{24}\right|+\left|\frac{4 y+x+2 y}{24}-\frac{4 v+u+2 v}{24}\right| \\
& \quad+\left|\frac{4 z+y+2 x}{24}-\frac{4 w+v+2 u}{24}\right| \\
& \leq \frac{7}{24}|x-u|+\frac{8}{24}|y-v|+\frac{6}{24}|z-w| .
\end{aligned}
$$

When $\varphi(t)=\frac{t}{2}$ and $g(x)=\frac{t}{2}$, we have

$$
\begin{aligned}
\frac{1}{4} & (|x-u|+|y-v|+|z-w|) \\
& =3 \frac{1}{2}\left(\frac{d(g(x), g(u))+d(g(y), g(v))+d(g(z), g(w))}{3}\right) \\
& =3 \varphi\left(\frac{d(g(x), g(u))+d(g(y), g(v))+d(g(z), g(w))}{3}\right) .
\end{aligned}
$$

It is easy to check that $F$ satisfies $(6)$ and $(0,0,0)$ is the tripled coincidence point of $F$.

If we take the mapping $g=I_{X}$ in Theorem 3.7 and Theorem 3.8, then we get the following.

Corollary 3.10 Let $(X, d)$ be a complete metric space and $M$ be a nonempty subset of $X^{6}$. Assume that there is a function $\varphi:[0, \infty) \rightarrow[0, \infty)$ with $0=\varphi(0)<\varphi(t)<t$ and $\lim _{r \rightarrow t^{+}} \varphi(r)<t$ for each $t>0$, and also suppose that $F: X \times X \times X \rightarrow X$ is a mapping such that

$$
\begin{aligned}
& d(F(x, y, z), F(u, v, w))+d(F(y, x, y), F(v, u, v))+d(F(z, y, x), F(w, v, u)) \\
& \quad \leq 3 \varphi\left(\frac{d(x, u)+d(y, v)+d(z, w)}{3}\right)
\end{aligned}
$$

for all $x, y, z, u, v, w \in X$ with $(x, y, z, u, v, w) \in M$ or $(u, v, w, x, y, z) \in M$. Suppose that either

(a) F is continuous, or

(b) iffor any three sequences $\left\{x_{n}\right\},\left\{y_{n}\right\},\left\{z_{n}\right\}$ with $\left(x_{n+1}, y_{n+1}, z_{n+1}, x_{n}, y_{n}, z_{n}\right) \in M$,

$\left\{x_{n}\right\} \rightarrow x,\left\{y_{n}\right\} \rightarrow y$ and $\left\{z_{n}\right\} \rightarrow z$ for all $n \geq 1$, then $\left(x, y, z, x_{n}, y_{n}, z_{n}\right) \in M$ for all $n \geq 1$.

If there exists $\left(x_{0}, y_{0}, z_{0}\right) \in X \times X \times X$ such that $\left(F\left(x_{0}, y_{0}, z_{0}\right), F\left(y_{0}, x_{0}, y_{0}\right), F\left(z_{0}, y_{0}, x_{0}\right), x_{0}\right.$, $\left.y_{0}, z_{0}\right) \in M$ and $M$ is an $F$-invariant set which satisfies the transitive property, then there exist $x, y, z \in X$ such that

$$
x=F(x, y, z), \quad y=F(y, x, y) \quad \text { and } \quad z=F(z, y, x) .
$$

Now we shall prove the uniqueness of a tripled fixed point. 
Theorem 3.11 In addition to the hypotheses of Theorem 3.7, suppose that for every $(x, y, z),\left(x^{*}, y^{*}, z^{*}\right) \in X \times X \times X$, there exists $(u, v, w) \in X \times X \times X$ such that $(g(x), g(y), g(z)$, $g(u), g(v), g(w)) \in M$ and $\left(g\left(x^{*}\right), g\left(y^{*}\right), g\left(z^{*}\right), g(u), g(v)\right), g(w) \in M$. Then $F$ and $g$ have a unique tripled common fixed point, that is, there exists a unique $(x, y, z) \in X \times X \times X$ such that

$$
x=g(x)=F(x, y, z), \quad y=g(y)=F(y, x, y) \quad \text { and } \quad z=g(z)=F(z, y, x) .
$$

Proof From Theorem 3.7, the set of tripled coincidence points is nonempty. Suppose $(x, y, z)$ and $\left(x^{*}, y^{*}, z^{*}\right)$ are tripled coincidence points of $F$, that is, $g(x)=F(x, y, z), g(y)=$ $F(y, x, y), g(z)=F(z, y, x), g\left(x^{*}\right)=F\left(x^{*}, y^{*}, z^{*}\right), g\left(y^{*}\right)=F\left(y^{*}, x^{*}, y^{*}\right)$ and $g\left(z^{*}\right)=F\left(z^{*}, y^{*}, x^{*}\right)$.

We shall show that

$$
g(x)=g\left(x^{*}\right), \quad g(y)=g\left(y^{*}\right) \quad \text { and } \quad g(z)=g\left(z^{*}\right) .
$$

By assumption, there is $(u, v, w) \in X \times X \times X$ such that $(g(x), g(y), g(z), g(u), g(v), g(w)) \in M$ and $\left(g\left(x^{*}\right), g\left(y^{*}\right), g\left(z^{*}\right), g(u), g(v), g(w)\right) \in M$.

Put $u_{0}=u, v_{0}=v, w_{0}=w$ and choose $u_{1}, v_{1}, w_{1} \in X$.

So that $g\left(u_{1}\right)=F\left(u_{0}, v_{0}, w_{0}\right), g\left(v_{1}\right)=F\left(v_{0}, u_{0}, v_{0}\right)$ and $g\left(w_{1}\right)=F\left(w_{0}, v_{0}, u_{0}\right)$. Then, similarly as in Theorem 3.7, we can inductively define sequences $\left\{g\left(u_{n}\right)\right\},\left\{g\left(v_{n}\right)\right\}$ and $\left\{g\left(w_{n}\right)\right\}$ such that

$$
\begin{gathered}
g\left(u_{n}\right)=F\left(u_{n-1}, v_{n-1}, w_{n-1}\right), \quad g\left(v_{n}\right)=F\left(v_{n-1}, u_{n-1}, v_{n-1}\right) \\
\text { and } g\left(w_{n}\right)=F\left(w_{n-1}, v_{n-1}, u_{n-1}\right) \quad \text { for all } n \geq 1 .
\end{gathered}
$$

It easy to show that

$$
\left(g(x), g(y), g(z), g\left(u_{n}\right), g\left(v_{n}\right), g\left(w_{n}\right)\right) \in M \quad \text { for all } n \geq 1 .
$$

Thus, from (6) and (27), we have

$$
\begin{aligned}
& \frac{d\left(g(x), g\left(u_{n+1}\right)\right)+d\left(g(y), g\left(v_{n+1}\right)\right)+d\left(g(z), g\left(w_{n+1}\right)\right)}{3} \\
& =\frac{d\left(F(x, y, z), F\left(u_{n}, v_{n}, w_{n}\right)\right)+d\left(F(y, x, y), F\left(v_{n}, u_{n}, v_{n}\right)\right)}{3} \\
& \quad+\frac{d\left(F(z, y, x), F\left(w_{n}, v_{n}, u_{n}\right)\right)+d\left(F(z, y, x), F\left(w_{n}, v_{n}, u_{n}\right)\right)}{3} \\
& \leq \varphi\left(\frac{d\left(g(x), g\left(u_{n}\right)\right)+d\left(g(y), g\left(v_{n}\right)\right)+d\left(g(z), g\left(w_{n}\right)\right)}{3}\right)
\end{aligned}
$$

for all $n \geq 1$. Thus, by (28), we deduce that the sequence $\left\{p_{n}\right\}$ is defined by

$$
p_{n}=\frac{d\left(g(x), g\left(u_{n}\right)\right)+d\left(g(y), g\left(v_{n}\right)\right)+d\left(g(z), g\left(w_{n}\right)\right)}{3} \text { for all } n \geq 1 \text {. }
$$

From (28) and (29), we have that $\left\{p_{n}\right\}$ is non-increasing. Hence, there exists $\alpha \geq 0$ such that

$$
\lim _{n \rightarrow \infty} p_{n}=\lim _{n \rightarrow \infty}\left(\frac{d\left(g(x), g\left(u_{n}\right)\right)+d\left(g(y), g\left(v_{n}\right)\right)+d\left(g(z), g\left(w_{n}\right)\right)}{3}\right)=\alpha .
$$


Suppose, to the contrary, that $\alpha>0$. Letting $n \rightarrow \infty$ in (28), we get

$$
\alpha \leq \lim _{n \rightarrow \infty} \varphi\left(p_{n}\right)=\lim _{p_{n} \rightarrow \alpha^{+}} \varphi\left(p_{n}\right)<\alpha,
$$

which is a contradiction. Thus $\alpha=0$, that is,

$$
\lim _{n \rightarrow \infty} p_{n}=\lim _{n \rightarrow \infty}\left(\frac{d\left(g(x), g\left(u_{n}\right)\right)+d\left(g(y), g\left(v_{n}\right)\right)+d\left(g(z), g\left(w_{n}\right)\right)}{3}\right)=0,
$$

which implies

$$
\lim _{n \rightarrow \infty} d\left(g(x), g\left(u_{n}\right)\right)=\lim _{n \rightarrow \infty} d\left(g(y), g\left(v_{n}\right)\right)=\lim _{n \rightarrow \infty} d\left(g(z), g\left(w_{n}\right)\right)=0 .
$$

Similarly, we obtain that

$$
\lim _{n \rightarrow \infty} d\left(g\left(x^{*}\right), g\left(u_{n}\right)\right)=\lim _{n \rightarrow \infty} d\left(g\left(y^{*}\right), g\left(v_{n}\right)\right)=\lim _{n \rightarrow \infty} d\left(g\left(z^{*}\right), g\left(w_{n}\right)\right)=0 .
$$

By (30) and (31) and the triangle inequality, we have

$$
\begin{aligned}
& d\left(g(x), g\left(x^{*}\right)\right) \leq d\left(g(x), g\left(u_{n+1}\right)\right)+d\left(g\left(u_{n+1}\right), g\left(x^{*}\right)\right), \\
& d\left(g(y), g\left(y^{*}\right)\right) \leq d\left(g(y), g\left(v_{n+1}\right)\right)+d\left(g\left(v_{n+1}\right), g\left(y^{*}\right)\right), \\
& d\left(g(z), g\left(z^{*}\right)\right) \leq d\left(g(z), g\left(w_{n+1}\right)\right)+d\left(g\left(w_{n+1}\right), g\left(z^{*}\right)\right) .
\end{aligned}
$$

Let $n \rightarrow \infty$ in (32). Hence $g\left(x^{*}\right)=g(x), g\left(y^{*}\right)=g(y)$ and $g\left(z^{*}\right)=g(z)$. Since $g(x)=F(x, y, z)$, $g(y)=F(y, x, y)$ and $g(z)=F(z, y, x)$, by commutativity of $F$ and $g$, we have

$$
\begin{aligned}
& g(g(x))=g(F(x, y, z))=F(g(x), g(y), g(z)), \\
& g(g(y))=g(F(y, x, y))=F(g(y), g(x), g(y)), \\
& g(g(z))=g(F(z, y, x))=F(g(z), g(y), g(x)) .
\end{aligned}
$$

Denote $a=g(x), b=g(y)$ and $c=g(z)$. Then from (33),

$$
g(a)=F(a, b, c), \quad g(b)=F(b, a, b) \quad \text { and } \quad g(c)=F(c, b, a) .
$$

Thus $(a, b, c)$ is a tripled coincidence point. Then from (26) with $x^{*}=a, y^{*}=b$ and $z^{*}=c$ it follows that $g(x)=g(a), g(y)=g(b)$ and $g(z)=g(c)$, that is,

$$
g(a)=a, \quad g(b)=b \quad \text { and } \quad g(c)=c .
$$

From (34) and (35), we have

$$
a=g(a)=F(a, b, c), \quad b=g(b)=F(b, a, b) \quad \text { and } \quad w=g(c)=F(c, b, a) .
$$

Therefore, $(a, b, c)$ is a tripled common fixed point of $F$ and $g$. To prove the uniqueness, assume that $(p, q, r)$ is another coupled common fixed point. Then by (26) we have

$$
p=g(p)=g(a)=a, \quad q=g(q)=g(b)=b \quad \text { and } \quad r=g(r)=g(c)=c .
$$


Next, we give a simple application of our results to tripled coincidence point theorems in partially ordered metric spaces.

Corollary 3.12 Let $(X, \leq)$ be a partially ordered set, and suppose that there is a metric $d$ on $X$ such that $(X, d)$ is a complete metric space. Assume that there is a function $\varphi$ : $[0, \infty) \rightarrow[0, \infty)$ with $\varphi(t)<t$ and $\lim _{r \rightarrow t^{+}} \varphi(r)<t$ for each $t>0$, and also suppose that $F: X \times X \times X \rightarrow X$ and $g: X \rightarrow X$ are such that $F$ has the mixed $g$-monotone property and

$$
\begin{aligned}
& d(F(x, y, z), F(u, v, w))+d(F(y, x, y), F(v, u, v))+d(F(z, y, x), F(w, v, u)) \\
& \quad \leq 3 \varphi\left(\frac{d(g(x), g(u))+d(g(y), g(v))+d(g(z), g(w))}{3}\right)
\end{aligned}
$$

for all $x, y, z, u, v, w \in X$ with $g(x) \leq g(u), g(y) \geq g(v)$ and $g(z) \leq g(w)$. Suppose that $F(X \times$ $X) \subseteq g(X), g$ is continuous and commutes with $F$ and $(g(X), d)$ is a complete metric space and $X$ has the following properties:

(i) if a non-decreasing sequence $\left\{x_{n}\right\} \rightarrow x$ in $X$, then $x_{n} \leq x$ for all $n$,

(ii) if a non-increasing sequence $\left\{y_{n}\right\} \rightarrow y$ in $X$, then $y \leq y_{n}$ for all $n$.

If there exist $x_{0}, y_{0}, z_{0} \in X$ such that

$$
g\left(x_{0}\right) \leq F\left(x_{0}, y_{0}, z_{0}\right), \quad g\left(y_{0}\right) \geq F\left(y_{0}, x_{0}, y_{0}\right) \quad \text { and } \quad g\left(z_{0}\right) \leq F\left(z_{0}, y_{0}, x_{0}\right),
$$

then there exist $x, y, z \in X$ such that

$$
g(x)=F(x, y, z), \quad g(y)=F(y, x, y) \quad \text { and } \quad g(z)=F(z, y, x) .
$$

Proof Define a subset $M \subseteq X^{6}$ by $M=\left\{(a, b, c, d, e, f) \in X^{6}: a \geq d, b \leq e, c \geq f\right\}$, then $M$ is an $(F, g)$-invariant set which satisfies the transitive property. By (36), we have

$$
\begin{aligned}
& d(F(x, y, z), F(u, v, w))+d(F(y, x, y), F(v, u, v))+d(F(z, y, x), F(w, v, u)) \\
& \quad \leq 3 \varphi\left(\frac{d(g(x), g(u))+d(g(y), g(v))+d(g(z), g(w))}{3}\right)
\end{aligned}
$$

for all $x, y, z, u, v, w \in X$ with $(g(x), g(y), g(z), g(u), g(v), g(w)) \in M$ or $(g(u), g(v), g(w), g(x)$, $g(y), g(z)) \in M$. Since $x_{0}, y_{0}, z_{0} \in X$ such that

$$
g\left(x_{0}\right) \leq F\left(x_{0}, y_{0}, z_{0}\right), \quad g\left(y_{0}\right) \geq F\left(y_{0}, x_{0}, y_{0}\right) \quad \text { and } \quad g\left(z_{0}\right) \leq F\left(z_{0}, y_{0}, x_{0}\right),
$$

we get $\left(F\left(x_{0}, y_{0}, z_{0}\right), F\left(y_{0}, x_{0}, y_{0}\right), F\left(z_{0}, y_{0}, x_{0}\right), g\left(x_{0}\right), g\left(y_{0}\right), g\left(z_{0}\right)\right) \in M$. For any three sequences $\left\{g\left(x_{n}\right)\right\},\left\{g\left(y_{n}\right)\right\},\left\{g\left(z_{n}\right)\right\}$ such that $\left\{g\left(x_{n}\right)\right\}$ is a non-decreasing sequence in $X$ with $\left\{g\left(x_{n}\right)\right\} \rightarrow x,\left\{g\left(y_{n}\right)\right\}$ is a non-increasing sequence in $X$ with $\left\{g\left(y_{n}\right)\right\} \rightarrow y$ and $\left\{g\left(z_{n}\right)\right\}$ is a non-decreasing sequence in $X$ with $\left\{g\left(z_{n}\right)\right\} \rightarrow z$, we have

$$
\begin{aligned}
& g\left(x_{1}\right) \leq g\left(x_{2}\right) \leq \cdots \leq g\left(x_{n}\right) \leq \cdots \leq x, \\
& g\left(y_{1}\right) \geq g\left(y_{2}\right) \geq \cdots \geq g\left(y_{n}\right) \geq \cdots \geq y
\end{aligned}
$$


and

$$
g\left(z_{1}\right) \leq g\left(z_{2}\right) \leq \cdots \leq g\left(z_{n}\right) \leq \cdots \leq z
$$

for all $n \geq 1$. Therefore, we have $\left(x, y, z, g\left(x_{n}\right), g\left(y_{n}\right), g\left(z_{n}\right)\right) \in M$ for all $n \geq 1$, and so the assumption of Theorem 3.8 holds, thus $F$ has a triple coincidence point.

Corollary 3.13 In addition to the hypotheses of Corollary 3.12, suppose that for all $(x, y, z),\left(x^{*}, y^{*}, z^{*}\right) \in X \times X \times X$, there exists $(u, v, w) \in X \times X \times X$ such that $g(x) \succeq g(u)$, $g(y) \preceq g(v), g(z) \succeq g(w)$ and $g\left(x^{*}\right) \succeq g(u), g\left(y^{*}\right) \preceq g(v), g\left(z^{*}\right) \succeq g(w)$. Then $F$ and $g$ have $a$ unique tripled common fixed point.

Proof Define a subset $M \subseteq X^{6}$ by $M=\left\{(a, b, c, d, e, f) \in X^{6}: a \geq d, b \leq e, c \geq f\right\}$, then $M$ is an $(F, g)$-invariant set which satisfies the transitive property. Thus, the proof of the existence of a tripled common fixed point is straightforward by following the same lines as in the proof of Corollary 3.12.

Next, we show the uniqueness of a tripled common fixed point. Since for all $(x, y, z),\left(x^{*}\right.$, $\left.y^{*}, z^{*}\right) \in X \times X \times X$, there exists $(u, v, w) \in X \times X \times X$ such that $g(x) \succeq g(u), g(y) \preceq$ $g(v), g(z) \succeq g(w)$ and $g\left(x^{*}\right) \succeq g(u), g\left(y^{*}\right) \preceq g(v), g\left(z^{*}\right) \succeq g(w)$, we can conclude that $(g(x), g(y), g(z), g(u), g(v), g(w)) \in M$ and $\left(g\left(x^{*}\right), g\left(y^{*}\right), g\left(z^{*}\right), g(u), g(v), g(w)\right) \in M$. Therefore, since all the hypotheses of Theorem 3.11 hold, $F$ and $g$ have a unique tripled common fixed point. This completes the proof.

Remark This research can be extended and improved to the more general case; see $[9$, 35-37].

\section{Competing interests}

The author declares that they have no competing interests.

\section{Authors' contributions}

The author read and approved the final manuscript.

\section{Acknowledgements}

This research was supported by Chiang Mai University and the author would like to express sincere appreciation to Prof. Suthep Suantai and the referees for very helpful suggestions and many kind comments.

Received: 28 June 2013 Accepted: 17 September 2013 Published: 07 Nov 2013

\section{References}

1. Ran, ACM, Reurings, MCB: A fixed point theorem in partially ordered sets and some applications to matrix equations. Proc. Am. Math. Soc. 132, 1435-1443 (2004)

2. Nieto, JJ, Rodriguez-Lopez, R: Contractive mapping theorems in partially ordered sets and applications to ordinary differential equation. Order 22, 223-239 (2005)

3. Nieto, JJ, Rodriguez-Lopez, R: Existence and uniqueness of fixed point in partially ordered sets and applications to ordinary differential equation. Acta Math. Sin. Engl. Ser. 23(12), 2205-2212 (2007)

4. Agarwal, RP, El-Gebeily, MA, O'Regan, D: Generalized contractions in partially ordered metric spaces. Appl. Anal. 87, $1-8(2008)$

5. Guo, D, Lakshmikantham, V: Coupled fixed points of nonlinear operators with applications. Nonlinear Anal. 11, 623-632 (1987)

6. Gnana Bhaskar, T, Lakshmikantham, V: Fixed point theorems in partially ordered metric spaces and applications. Nonlinear Anal. TMA 65, 1379-1393 (2006)

7. Alsulami, SM, Alotaibi, A: Tripled coincidence points for monotone operators in partially ordered metric spaces. Int. Math. Forum 7, 1811-1824 (2012)

8. Mursaleen, M, Mohiuddine, SA, Agawal, RP: Coupled fixed point theorems for $\alpha$ - $\psi$-contractive type mappings in partially ordered metric spaces. Fixed Point Theory Appl. 2012, 228 (2012) [Corrigendum to Coupled fixed point theorems for $\alpha-\psi$-contractive type mappings in partially ordered metric spaces. Fixed Point Theory Appl. 2013, 127 (2013)] 
9. Berzig, M, Samet, B: An extension of coupled fixed point's concept in higher dimension and applications. Comput. Math. Appl. 63, 1319-1334 (2012)

10. Samet, B, Vetro, C: Coupled fixed point F-invariant set and fixed point of $N$-order. Ann. Funct. Anal. 1, 46-56 (2010)

11. Amini-Harandi, A: Coupled and tripled fixed point theory in partially ordered metric spaces with application to initial value problem. Math. Comput. Model. 57(910), 2343-2348 (2013)

12. Hussain, N, Latif, A, Shah, MH: Coupled and tripled coincidence point results without compatibility. Fixed Point Theory Appl. 2012, 77 (2012)

13. Berinde, V: Coupled fixed point theorems for $\varphi$-contractive mixed monotone mappings in partially ordered metric spaces. Nonlinear Anal., Theory Methods Appl. 75(6), 3218-3228 (2012)

14. Berinde, V: Coupled coincidence point theorems for mixed monotone nonlinear operators. Comput. Math. Appl. 64(6), 1770-1777 (2012)

15. Sintunavarat, W, Kumam, P, Cho, YJ: Coupled fixed point theorems for nonlinear contractions without mixed monotone property. Fixed Point Theory Appl. 2012, 170 (2012)

16. Sintunavarat, W, Radenović, S, Golubović, Z, Kuman, P: Coupled fixed point theorems for F-invariant set. Appl. Math. Inform. Sci. 7(1), 247-255 (2013)

17. Batra, R, Vashistha, S: Coupled coincidence point theorems for nonlinear contractions under $(F, g)$-invariant set in cone metric spaces. J. Nonlinear Sci. Appl. 6, 86-96 (2013)

18. Lakshmikantham, V, Ćirić, L: Coupled fixed point theorems for nonlinear contractions in partially ordered metric spaces. Nonlinear Anal. TMA 70, 4341-4349 (2009)

19. Luong, NV, Thuan, NX: Coupled fixed points in partially ordered metric spaces and application. Nonlinear Anal. 74 983-992 (2011)

20. Berinde, V, Borcut, M: Tripled fixed point theorems for contractive type mappings in partially ordered metric spaces. Nonlinear Anal. 74, 4889-4897 (2011)

21. Berinde, $\mathrm{V}$, Borcut, $\mathrm{M}$ : Tripled coincidence theorems for contractive type mappings in partially ordered metric spaces, Appl. Math. Comput. 218(10), 5929-5936 (2010)

22. Abbas, M, Aydi, H, Karapinar, E: Tripled fixed points of multi-valued nonlinear contraction mappings in partially ordered metric spaces. Abstr. Appl. Anal. 2011, Article ID 812690 (2011)

23. Abbas, M, Ali, B, Sintunavarat, W, Kumam, P: Tripled fixed point and tripled coincidence point theorems in intuitionistic fuzzy normed spaces. Fixed Point Theory Appl. 2012, Article ID 187 (2012)

24. Aydi, H, Karapinar, E, Postolache, M: Tripled coincidence points for weak $\phi$-contractions in partially ordered metric spaces. Fixed Point Theory Appl. 2012, 44 (2012)

25. Aydi, H, Karapinar, E, Vetro, C: Meir-Keeler type contractions for tripled fixed points. Acta Math. Sci. 32(6), 2119-2130 (2012)

26. Aydi, H, Karapinar, E: New Meir-Keeler type tripled fixed point theorems on ordered partial metric spaces. Math. Probl. Eng. 2012, Article ID 409872 (2012)

27. Aydi, H, Abbas, $M$, Sintunavarat, $W$, Kumam, P: Tripled fixed point of $W$-compatible mappings in abstract metric spaces. Fixed Point Theory Appl. 2012, 134 (2012)

28. Aydi, H, Abbas, M: Tripled coincidence and fixed point results in partial metric spaces. Appl. Gen. Topol. 13(2), 193-206 (2012)

29. Aydi, H, Karapinar, E: Tripled fixed points in ordered metric spaces. Bull. Math. Anal. Appl. 4(1), 197-207 (2012)

30. Aydi, H, Karapinar, E, Shatanawi, W: Tripled coincidence point results for generalized contractions in ordered generalized metric spaces. Fixed Point Theory Appl. 2013, 34 (2013)

31. Borcut, M: Tripled coincidence theorems for contractive type mappings in partially ordered metric spaces. Appl. Math. Comput. 218, 7339-7346 (2012)

32. Choudhury, BS, Karapinar, E, Kundu, A: Tripled coincidence point theorems for nonlinear contractions in partially ordered metric spaces. Int. J. Math. Math. Sci. 2012, Article ID 329298 (2012)

33. Charoensawan, P: Tripled fixed points theorems for $\phi$-contractive mixed monotone operators on partially ordered metric spaces. Appl. Math. Sci. 6, 5229-5239 (2012)

34. Mohiuddine, SA, Alotaibi, A: Some results on a tripled fixed point for nonlinear contractions in partially ordered G-metric spaces. Fixed Point Theory Appl. 2012, 179 (2012)

35. Roldan, A, Martinez-Moreno, J, Roldan, C: Multidimensional fixed point theorems in partially ordered complete metric spaces. J. Math. Anal. Appl. 396(2), 536-545 (2012)

36. Roldan, A, Martinez-Moreno, J, Roldan, C, Karapinar, E: Multidimensional fixed point theorems in partially ordered complete partial metric spaces under $(\psi, \varphi)$-contractivity conditions. Abstr. Appl. Anal. 2013, 634371 (2013)

37. Karapinar, E, Roldan, A, Martinez-Moreno, J, Roldan, C: Meir-Keeler type multidimensional fixed point theorems in partially ordered metric spaces. Abstr. Appl. Anal. 2013, 406026 (2013) 